A C G

Org. Commun. 14:1 (2021) 48-57

\title{
organic
}

publications

communications

\section{One-pot protocol for the synthesis of quinoxalines from styrenes, o-phenylenediamine and benzo[c] $][1,2,5]$ thiadiazole-4,5-diamine using triiodoisocyanuric acid}

\author{
Suresh Kuarm Bowroju $\oplus^{1}$ Hanumaiah Marumamula $\oplus^{2}$ \\ and Rajitha Bavanthula 1* $^{*}$

\begin{abstract}
${ }^{2}$ Organic and Biomolecular Chemistry Division, CSIR-Indian Institute of Chemical Technology, Hyderabad, India
\end{abstract} \\ ${ }^{1}$ Department of Chemistry, National Institution Technology, Warangal, Telangana, 506 004, India
}

(Received September 08, 2020; Revised January 01, 2021; Accepted January 20, 2021)

\begin{abstract}
Triiodoisocyanuric acid (TICA) controlled one-pot and easy-operational protocol has been developed for the synthesis of substituted phenylquinoxalines (3a-3i) and phenyl-[1,2,5]thiadiazolo[3,4-f]quinoxaline (5a5f) from styrenes with o-phenylenediamine and benzo[c][1,2,5]thiadiazole-4,5-diamine respectively. The reaction involves co-bromination and oxidation for the formation of an a-bromo ketone as an intermediate in the presence of triiodoisocyanuric acid, followed by condensation with the o-phenylenediamine and benzo[c][1,2,5]thiadiazole-4,5-diamine for the formation of phenylquinoxalines (3a-3i) and phenyl$[1,2,5]$ thiadiazolo[3,4-f]quinoxaline (5a-5f) in 55-79\% yield. This protocol environmentally benign and economically viable. Substituted quinoxalines were obtained in good to excellent yields with wide substrate scope and functional-group tolerance.
\end{abstract}

Keywords: Quinoxalines; one-pot protocol; triiodoisocyanuric acid; styrenes. C2021 ACG Publication. All right reserved.

\section{Introduction}

Quinoxalines are ubiquitous motifs, which have found in a variety of natural products and synthetic pharmaceuticals, many of which exhibit favorable biological activities (Figure1) and has always been an attractive heterocycle to biochemists because of its valuable contribution in biological sciences $^{1}$. In particular, many of these quinoxaline derivatives have exhibit wide range of biological activities, such as antimalarial ${ }^{2}$ antitumour $^{3}$, anticancer ${ }^{4}$, antiproliferative ${ }^{5}$ and antileishmanial ${ }^{6}$. Quinoxaline derivatives play an important role in amyloid fibril detection owing to their outstanding fluorescence properties ${ }^{7}$. As of now, several methods have been proposed as a common method to afford simple and substituted quinoxalines by oxidative coupling ${ }^{8}, \mathrm{MnO}_{2}{ }^{9}, \mathrm{POCl}_{3}{ }^{10}$, ceric ammonium nitrate ${ }^{11}$, iodine ${ }^{12}$, montmorillonite $\mathrm{K}-10^{13}, \mathrm{Ga}(\mathrm{OTf})_{3}{ }^{14}$, silica gel ${ }^{15}$, citric acid $^{16}$, Oxalic acid/EtOH ${ }^{17}$, sulfated $\mathrm{TiO}_{2}{ }^{18}$, sulfated $\mathrm{TiO}_{2}-\mathrm{P} 25{ }^{19}$, acetic acid/copper catalyzed oxidative cyclization of a-hydroxy ketones with 1,2-diamines ${ }^{20}$ and cellulose sulfuric acid ${ }^{21}$. Nevertheless, most of the existing methodologies suffer from one or more disadvantages in the process of synthesis which are

* Corresponding author: E-Mail: rajithabhargavi@ymail.com 
unsatisfactory yields, longer reaction time, difficulties procedure to this scaffold is still valuable synthesis of quinoxaline.

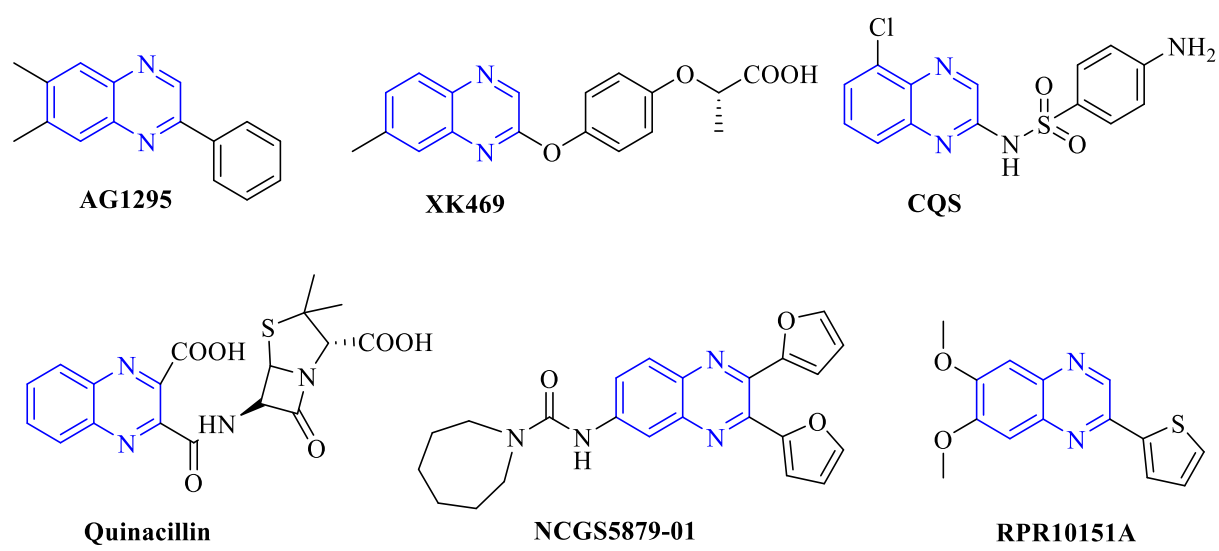

Figure 1. Quinoxaline cored natural and bioactive compounds.

In the product isolation process, moreover the use of highly expensive and detrimental metal precursors, drastic reaction conditions were observed and also no agreement with the green chemistry protocols, which limit their use. Hence it is highly essential to develop a convenient, economical and environmentally benign synthesis this context, one pot tactics are widespread in the literature for the synthesis of heterocycles using $\mathrm{N}$-halo reagents ${ }^{22-23}$. Among the $\mathrm{N}$-halo reagents, especially trihaloisocyanuric acids are commercially available or easily prepared from available chemicals. This trihaloisocyanuric acids are most effective and stable electrophilic halogenating reagents ${ }^{24-25}$ and are able to transfer up to three halogen atoms to a substrate ${ }^{26}$. In addition, by-products formed from the reaction using trihaloisocyanuric acids can be reusable to produce further trihaloisocyanuric acid ${ }^{27}$. In continuation our interest on methodologies ${ }^{28-48}$, we report herein the synthesis of quinoxalines using $\mathrm{TICA}^{49}$ as a catalyst promoted conversion of styrenes into quinoxalines through a one-pot approach of three consecutive reactions.

\section{Experimental}

\subsection{General}

Melting points are uncorrected and were determined in open capillaries. The reactions were monitored by thin layer chromatography (TLC) and visualized with UV light. NMR spectra on Agilent $400 \mathrm{MHz}$ spectrometer using TMS as an internal standard. Spectral analyses were carried out in $\mathrm{CDCl}_{3}$ or DMSO- $d_{6}$ for both ${ }^{1} \mathrm{H}$ and ${ }^{13} \mathrm{C}$ spectra. Chemical shifts were measured in $\delta$ parts per million (ppm) and coupling constants $(J)$ were measured in hertz $(\mathrm{Hz})$. All solvents and reagents were purchased from Aldrich.

\subsection{General Procedure for the Synthesis of Quinoxalines}

A round-bottom flask equipped with a magnetic stirring bar was charged with styrene (1) (1.0 mmol), TICA $(3.0 \mathrm{mmol})$ and water $(2.0 \mathrm{~mL})$ at room temperature. The resulting mixture was heated to $80{ }^{\circ} \mathrm{C}$ for $1 \mathrm{~h}$. Then, acetone was added and the reaction media was filtered, to this filtrate ophenylenediamine $(1 \mathrm{mmol})$ or benzo[c][1,2,5]thiadiazole-4,5-diamine $(1 \mathrm{mmol})$ was added and stirred at room temperature for $30 \mathrm{~min}$. After completion of the reaction (monitored by TLC), the reaction mixture was poured over ice water, neutralized with $\mathrm{NH}_{4} \mathrm{OH}$ and the precipitated solid was filtered off and recrystallized from $\mathrm{EtOH}$ to afford the corresponding pure products. 
2-phenylquinoxaline $(3 a)^{50}$ : Solid; $71 \%$ Yield; m. $76-79{ }^{\circ} \mathrm{C}$. (Lit. m. $\left.75-78{ }^{\circ} \mathrm{C}\right) ;{ }^{1} \mathrm{H}$ NMR $(400 \mathrm{MHz}$, $\left.\mathrm{CDCl}_{3}\right): 9.28(\mathrm{~s}, 1 \mathrm{H}), 7.74-8.16(\mathrm{~m}, 4 \mathrm{H}), 7.73-7.66(\mathrm{~m}, 2 \mathrm{H}), 7.47-7.54(\mathrm{~m}, 3 \mathrm{H}) \mathrm{ppm},{ }^{13} \mathrm{C}$ NMR $(100$ $\left.\mathrm{MHz}, \mathrm{CDCl}_{3}\right): \delta 151.75,143.3,142.26,141.56,136.73,130.21,130.14,129.6,129.47,129.1,127.51$ ppm. EIMS, $70 \mathrm{ev}, m / z: 207.3(\mathrm{M}+\mathrm{H})^{+}$.

6-fluoro-2-phenylquinoxaline $(3 \boldsymbol{b})^{50}$ : Solid; $76 \%$ Yield; m. $118-120{ }^{\circ} \mathrm{C}$. (Lit. m. $\left.120-122{ }^{\circ} \mathrm{C}\right) ;{ }^{1} \mathrm{H}$ NMR (400 MHz, $\left.\mathrm{CDCl}_{3}\right): \delta 9.47(\mathrm{~s}, 1 \mathrm{H}), 9.01(\mathrm{~s}, 1 \mathrm{H}), 8.54(\mathrm{~d}, J=6.0 \mathrm{~Hz}, 1 \mathrm{H}), 8.28(\mathrm{~s}, 1 \mathrm{H}), 8.23-8.27$ $(\mathrm{m}, 2 \mathrm{H}), 7.57-7.59(\mathrm{~m}, 3 \mathrm{H}) \mathrm{ppm},{ }^{13} \mathrm{C}$ NMR $\left(100 \mathrm{MHz}, \mathrm{CDCl}_{3}\right): \delta$ 145.46. 131.37, 131.51, 129.39, $1273.91,125.64,123.75 \mathrm{ppm}$. EIMS, $70 \mathrm{ev}, \mathrm{m} / \mathrm{z}: 242.3(\mathrm{M}+\mathrm{H})^{+}$.

6-chloro-2-phenylquinoxaline $(3 \mathrm{c})^{50}$ : Solid; $74 \%$ Yield; m. $142-143{ }^{\circ} \mathrm{C}$. (Lit. m. $\left.142-144{ }^{\circ} \mathrm{C}\right) ;{ }^{1} \mathrm{H}$ $\operatorname{NMR}\left(400 \mathrm{MHz}, \mathrm{CDCl}_{3}\right): 9.24(\mathrm{~s}, 1 \mathrm{H}), 8.14(\mathrm{~d}, J=8.0 \mathrm{~Hz}, 2 \mathrm{H}), 8.08(\mathrm{~s}, 1 \mathrm{H}), 7.99(\mathrm{~d}, J=8.8 \mathrm{~Hz}, 1 \mathrm{H})$, $7.63(\mathrm{~d}, J=8.8 \mathrm{~Hz}, 1 \mathrm{H}), 7.47-7.54(\mathrm{~m}, 3 \mathrm{H}) \mathrm{ppm},{ }^{13} \mathrm{C} \mathrm{NMR}\left(100 \mathrm{MHz}, \mathrm{CDCl}_{3}\right): \delta 152.4,143.32,142.55$, $140.02,136.22,136.01,130.5,130.41,130.29,129.15,128.44,127.58$ ppm. EIMS, 70 ev, $m / z: 225.3$ $(\mathrm{M}+\mathrm{H})^{+}$.

2-(2-fluorophenyl)quinoxaline $(3 \boldsymbol{d})^{51}$ :Solid; $72 \%$ Yield; m. 60-62 ${ }^{\circ} \mathrm{C}$. (Lit. m. 60-61 $\left.{ }^{\circ} \mathrm{C}\right) ;{ }^{1} \mathrm{H}$ NMR $\left(400 \mathrm{MHz}, \mathrm{CDCl}_{3}\right): 9.22$ (s, $\left.1 \mathrm{H}\right), 8.14$ (t, $\left.J=9.2 \mathrm{~Hz}, 2 \mathrm{H}\right), 8.03$ (d, $\left.J=8.8 \mathrm{~Hz}, 1 \mathrm{H}\right), 7.69$ (d, $J=8.8 \mathrm{~Hz}$, $1 \mathrm{H}), 7.43-7.53(\mathrm{~m}, 4 \mathrm{H}) \mathrm{ppm},{ }^{13} \mathrm{C}$ NMR $\left(100 \mathrm{MHz}, \mathrm{CDCl}_{3}\right): \delta 161.73,152.36,143.18,138.74,136.31$, $131.17,131.07,130.43,129.13,127.55,119.89,113.1 \mathrm{ppm}$. EIMS, $70 \mathrm{ev}, \mathrm{m} / z: 226.2(\mathrm{M}+\mathrm{H})^{+}$.

2-(3-(trifluoromethyl)phenyl)quinoxaline $(3 \boldsymbol{e})^{52}$ : Solid; $70 \%$ Yield; m. 118-120 ${ }^{\circ} \mathrm{C}$. (Lit. m. 119$\left.121^{\circ} \mathrm{C}\right) 119-121 ;{ }^{1} \mathrm{H}$ NMR $\left(400 \mathrm{MHz}, \mathrm{CDCl}_{3}\right): 9.39$ (s, 1H), 8.40 (s, 1H), 8.19-8.25 (m, 3H), 7.59 (d, $J=8.8 \mathrm{~Hz}, 1 \mathrm{H}), 7.54-7.59(\mathrm{~m}, 3 \mathrm{H}) \mathrm{ppm},{ }^{13} \mathrm{C} \mathrm{NMR}\left(100 \mathrm{MHz}, \mathrm{CDCl}_{3}\right): \delta 153.5,144.61,140.53,136.02$, $130.85,130.8,129.26,127.72,127.19, .127 .15,125.94,125.91 \mathrm{ppm}$. EIMS, $70 \mathrm{ev}, m / z: 275.3(\mathrm{M}+\mathrm{H})^{+}$.

6-bromo-5-fluoro-2-phenylquinoxaline (3f): Solid; $74 \%$ Yield; m. 130-132 ${ }^{\circ} \mathrm{C} ;{ }^{1} \mathrm{H}$ NMR $(400 \mathrm{MHz}$, $\left.\mathrm{CDCl}_{3}\right): 9.35(\mathrm{~s}, 1 \mathrm{H}), 8.17-8.19(\mathrm{~m}, 2 \mathrm{H}), 7.87(\mathrm{~d}, J=8.0 \mathrm{~Hz}, 1 \mathrm{H}), 7.73(\mathrm{~d}, J=8.4 \mathrm{~Hz}, 1 \mathrm{H}), 7.53-7.58(\mathrm{~m}$, $3 \mathrm{H}) \mathrm{ppm},{ }^{13} \mathrm{C}$ NMR $\left(100 \mathrm{MHz}, \mathrm{CDCl}_{3}\right): \delta 153.07,143.06,143.03,135.67,130.91,129.29,127.73$, 123.61, 123.32, 113.19, 112.98 ppm. EIMS, $70 \mathrm{ev}, \mathrm{m} / z: 303.2(\mathrm{M}+\mathrm{H})^{+}$.

2-(3-bromo-5-(trifluoromethyl)phenyl)quinoxaline (3g):Solid; $79 \%$ Yield; m. 102-104 ${ }^{\circ} \mathrm{C} ;{ }^{1} \mathrm{H}$ NMR (400 MHz, $\left.\mathrm{CDCl}_{3}\right): 9.43(\mathrm{~s}, 1 \mathrm{H}), 8.37(\mathrm{~s}, 1 \mathrm{H}), 8.33(\mathrm{~d}, J=8.0 \mathrm{~Hz}, 2 \mathrm{H}), 8.24(\mathrm{~s}, 1 \mathrm{H}), 7.57-7.59(\mathrm{~m}, 3 \mathrm{H})$ ppm, ${ }^{13} \mathrm{C}$ NMR $\left(100 \mathrm{MHz}, \mathrm{CDCl}_{3}\right): \delta 153.72,144.82,141.23,135.43,131.35,129.43,129.4,129.36$, $127.96,126.82,126.78,125.96$ ppm. EIMS, $70 \mathrm{ev}, \mathrm{m} / z: 352.9(\mathrm{M}+\mathrm{H})^{+}$.

6-bromo-2-phenylquinoxaline $(3 \boldsymbol{h})^{51}$ : Solid; $66 \%$ Yield; m. $128-130{ }^{\circ} \mathrm{C}$. (Lit. m. $\left.132-134{ }^{\circ} \mathrm{C}\right) ;{ }^{1} \mathrm{H}$ $\operatorname{NMR}\left(400 \mathrm{MHz}, \mathrm{CDCl}_{3}\right): 9.27(\mathrm{~s}, 1 \mathrm{H}), 8.28(\mathrm{~s}, 1 \mathrm{H}), 8.15(\mathrm{~d}, J=6.0 \mathrm{~Hz}, 2 \mathrm{H}), 7.93(\mathrm{~d}, J=8.8 \mathrm{~Hz}, 1 \mathrm{H})$, $7.77(\mathrm{~d}, J=8.8 \mathrm{~Hz}, 1 \mathrm{H}), 7.47-7.54(\mathrm{~m}, 3 \mathrm{H}) \mathrm{ppm},{ }^{13} \mathrm{C} \mathrm{NMR}\left(100 \mathrm{MHz}, \mathrm{CDCl}_{3}\right): \delta 152.36,143.47,142.84$, $140.27,136.21,132.98,131.84,130.52,129.17,127.56,124.24$ ppm. EIMS, $70 \mathrm{ev}, m / z: 285.1(\mathrm{M}+\mathrm{H})^{+}$.

2-(3-chlorophenyl)quinoxaline (3i) $)^{53}$ : Solid; $76 \%$ Yield; m. $130-132{ }^{\circ} \mathrm{C}$. (Lit. m. $\left.132-134{ }^{\circ} \mathrm{C}\right) ;{ }^{1} \mathrm{H}$ NMR (400 MHz, $\left.\mathrm{CDCl}_{3}\right): 9.53(\mathrm{~s}, 1 \mathrm{H}), 8.59(\mathrm{~s}, 1 \mathrm{H}), 8.30-8.32(\mathrm{~m}, 2 \mathrm{H}), 8.27(\mathrm{~s}, 1 \mathrm{H}), 7.57-7.59(\mathrm{~m}$, $3 \mathrm{H}) \mathrm{ppm} ;{ }^{13} \mathrm{C}$ NMR $\left(100 \mathrm{MHz}, \mathrm{CDCl}_{3}\right): \delta 153.34,144.94,140.7,135.2,131.59,131.35,131.3$, $131.26,129.44,129.39,128.0,127.73,124.5$ ppm. EIMS, $70 \mathrm{ev}, \mathrm{m} / z: 242.3(\mathrm{M}+\mathrm{H})^{+}$.

7-phenyl-[1,2,5]thiadiazolo[3,4-f]quinoxaline (5a): Solid; $68 \%$ Yield; m. 140-142 ${ }^{\circ} \mathrm{C} ;{ }^{1} \mathrm{H}$ NMR (400 $\left.\mathrm{MHz}, \mathrm{CDCl}_{3}\right): 9.37(\mathrm{~s}, 1 \mathrm{H}), 8.21-8.18(\mathrm{~m}, 2 \mathrm{H}), 7.88(\mathrm{~d}, J=8.0 \mathrm{~Hz}, 1 \mathrm{H}), 7.79(\mathrm{~d}, J=8.4 \mathrm{~Hz}, 1 \mathrm{H}), 7.26-$ $7.60(\mathrm{~m}, 3 \mathrm{H}) \mathrm{ppm},{ }^{13} \mathrm{C}$ NMR $\left(100 \mathrm{MHz}, \mathrm{CDCl}_{3}\right): \delta 155.31,145.30,145.27,137.91,133.15,131.53$, 129.97, 125.85, 125.56 115.43, 115.22 ppm. EIMS, $70 \mathrm{ev}, \mathrm{m} / z$ : $265.3(\mathrm{M}+\mathrm{H})^{+}$. Chemical Formula: $\mathrm{C}_{14} \mathrm{H}_{8} \mathrm{~N}_{4} \mathrm{~S}$ Elemental Analysis: C, 63.62; H, 3.05; N, 21.20; S, 12.13.

7-(2-chlorophenyl)-[1,2,5] thiadiazolo[3,4-f]quinoxaline (5b): Solid; $70 \%$ Yield; m. $116-118{ }^{\circ} \mathrm{C} ;{ }^{1} \mathrm{H}$ NMR (400 MHz, DMSO-d 6 ): 9.48 (s, 1H), 8.09 (d, $J=8.8 \mathrm{~Hz}, 1 \mathrm{H}), 7.78$ (d, $J=8.8 \mathrm{~Hz}, 1 \mathrm{H}), 7.69-7.71$ 
(m, 1H) 7.53-7.55 (m, 3H) ppm, ${ }^{13} \mathrm{C}$ NMR (100 MHz, DMSO-d 6 ): $\delta 164.07,161.59,152.07,143.59$, 143.56, 142.74, 142.6, 138.83, 136.09, 131.83, 131.73, 131.09, 129.52, 127.98, 120.46, 120.2, 113.1, 112.89 ppm. EIMS, $70 \mathrm{ev}, \mathrm{m} / \mathrm{z}: 299.4(\mathrm{M}+\mathrm{H})^{+}$. Chemical Formula: $\mathrm{C}_{14} \mathrm{H}_{7} \mathrm{ClN}_{4} \mathrm{~S}$ Elemental Analysis: , $56.28 ; \mathrm{H}, 2.36 ; \mathrm{Cl}, 11.87 ; \mathrm{N}, 18.75 ; \mathrm{S}, 10.73$.

7-(3-trifluoromethyl)-[1,2,5] thiadiazolo[3,4-f] quinoxaline (5c): Solid; $75 \%$ Yield; m. $168-170{ }^{\circ} \mathrm{C} ;{ }^{1} \mathrm{H}$ NMR (400 MHz, DMSO-d $)$ ): $9.53(\mathrm{~s}, 1 \mathrm{H}), 8.12(\mathrm{~s}, 1 \mathrm{H}), 8.05(\mathrm{~d}, J=8.8 \mathrm{~Hz}, 1 \mathrm{H}), 7.8(\mathrm{~d}, J=8.8 \mathrm{~Hz}, 1 \mathrm{H})$, 7.54-7.57 (m, 3H) ppm, ${ }^{13} \mathrm{C}$ NMR (100 MHz, DMSO-d 6 ): $\delta 152.24,144.57,142.23,140.12,136.01$, 135.33, 131.18, 131.1, 130.81, 129.56, 128.26, 128.04 ppm. EIMS, 70 ev, m/z: $333.2(\mathrm{M}+\mathrm{H})^{+}$. Chemical Formula: $\mathrm{C}_{15} \mathrm{H}_{7} \mathrm{~F}_{3} \mathrm{~N}_{4} \mathrm{~S}$ Elemental Analysis: C, 54.22; H, 2.12; F, 17.15; N, 16.86; S, 9.65.

7-(3-bromo-2-fluorophenyl)-[1,2,5]thiadiazolo[3,4-f]quinoxaline (5d): Solid; $62 \%$ Yield; m. 80$82^{\circ} \mathrm{C} ;{ }^{1} \mathrm{H}$ NMR $\left(400 \mathrm{MHz}, \mathrm{CDCl}_{3}\right)$ : $9.32(\mathrm{~s}, 1 \mathrm{H}), 7.90(\mathrm{~d}, J=8.0 \mathrm{~Hz}, 1 \mathrm{H}), 7.31(\mathrm{~d}, J=8.4 \mathrm{~Hz}, 1 \mathrm{H}), 7.53-$ $7.58(\mathrm{~m}, 3 \mathrm{H}) \mathrm{ppm},{ }^{13} \mathrm{C}$ NMR $\left(100 \mathrm{MHz}, \mathrm{CDCl}_{3}\right): \delta 144.31,130.69,129.24,127.56,124.39,124.1$, $112.78,112.57 \mathrm{ppm}$. EIMS, $70 \mathrm{ev}, \mathrm{m} / z: 361.4(\mathrm{M}+\mathrm{H})^{+}$. Chemical Formula: $\mathrm{C}_{14} \mathrm{H}_{6} \mathrm{BrFN}_{4} \mathrm{~S}$ Elemental Analysis: C, 46.55; H, 1.67; Br, 22.12; F, 5.26; N, 15.51; S, 8.88.

7-(2,3-dichlorophenyl)-[1,2,5] thiadiazolo[3,4-f]quinoxaline (5e): Solid; $65 \%$ Yield; m. 150-152 ${ }^{\circ} \mathrm{C}$; ${ }^{1} \mathrm{H}$ NMR (400 MHz, DMSO-d 6 ): 9.56 (s, 1H), 8.02 (d, $\left.J=8.8 \mathrm{~Hz}, 1 \mathrm{H}\right), 7.73$ (d, $\left.J=8.8 \mathrm{~Hz}, 1 \mathrm{H}\right), 7.55-$ $7.58(\mathrm{~m}, 3 \mathrm{H}) \mathrm{ppm},{ }^{13} \mathrm{C}$ NMR $\left(100 \mathrm{MHz}, \mathrm{DMSO}-\mathrm{d}_{6}\right): \delta 152.21,144.72,142.55,140.34,136.03,133.4$, 131.56, 131.21, 131.18, 129.59, 128.06, $124.02 \mathrm{ppm}$. EIMS, $70 \mathrm{ev}, \mathrm{m} / z: 332.9(\mathrm{M}+\mathrm{H})^{+}$. Chemical Formula: $\mathrm{C}_{14} \mathrm{H}_{6} \mathrm{Cl}_{2} \mathrm{~N}_{4} \mathrm{~S}$ Elemental Analysis: $\mathrm{C}, 50.47 ; \mathrm{H}, 1.82 ; \mathrm{Cl}, 21.28 ; \mathrm{N}, 16.82 ; \mathrm{S}, 9.62$.

7-(2,3-difluorophenyl)-[1,2,5] thiadiazolo[3,4-f]quinoxaline (5f): Solid; $67 \%$ Yield; m. 156-160 ${ }^{\circ} \mathrm{C}$; ${ }^{1} \mathrm{H}$ NMR (400 MHz, $\left.\mathrm{CDCl}_{3}\right): 9.48(\mathrm{~s}, 1 \mathrm{H}), 8.59$ (d, $\left.J=8.8 \mathrm{~Hz}, 1 \mathrm{H}\right), 7.92(\mathrm{~d}, J=8.8 \mathrm{~Hz}, 1 \mathrm{H}), 7.59-7.61$ $(\mathrm{m}, 3 \mathrm{H}) \mathrm{ppm},{ }^{13} \mathrm{C} \mathrm{NMR}\left(100 \mathrm{MHz}, \mathrm{CDCl}_{3}\right): \delta 152.21,144.72,142.55,140.34,136.03,133.4,131.56$, 131.21, 131.18, 129.59, 128.06, 124.02 ppm. EIMS, $70 \mathrm{ev}, \mathrm{m} / \mathrm{z}: 301.1(\mathrm{M}+\mathrm{H})^{+}$. Chemical Formula: $\mathrm{C}_{14} \mathrm{H}_{6} \mathrm{~F}_{2} \mathrm{~N}_{4} \mathrm{~S}$ Elemental Analysis: C, 56.00; H, 2.01; F, 12.65; N, 18.66; S, 10.68 .

\section{Results and Discussion}

We started the initial study of the one-pot tandem reaction, the direct conversion of styrene into phenacyl iodide through with excess TICA having a double action, i.e. as an electrophilic halogen source to form the bromohydrin and an oxidant to convert it into the haloketone ${ }^{54}$. As shown Table 1 , the reaction with styrene and TICA afforded the desired phenacyl iodide in $25 \%$ yield when it was conducted in acetone: $\mathrm{H}_{2} \mathrm{O}(1: 1)$ at $\mathrm{rt}$ for $1 \mathrm{~h}$ (Entry 1). To increase the yield of the product, several conditions were tested. The reactions in different solvents at different temperatures by increasing or decreasing time. Finally, when the reaction was carried out in acetone, $\mathrm{H}_{2} \mathrm{O}(2: 1)$ at $\mathrm{rt}$ for $15 \mathrm{~h}$, the desired product was formed in $100 \%$ yield (Entry 3 ) and when the reaction was carried out $\mathrm{H}_{2} \mathrm{O}$ alone at $80{ }^{\circ} \mathrm{C}$ for $1 \mathrm{~h}$, the desired product was formed in $100 \%$ yield (Entry 7 ).

Table 1. Optimizations of reaction conditions

\begin{tabular}{lrrrc}
\hline Solvent & Equiv. & \multicolumn{1}{c}{ Time } & Temp. & Yield \\
\hline $\mathrm{Me}_{2} \mathrm{CO}: \mathrm{H}_{2} \mathrm{O}(1: 1)$ & 3.0 & $1 \mathrm{~h}$ & r.t & 25 \\
$\mathrm{Me}_{2} \mathrm{CO}: \mathrm{H}_{2} \mathrm{O}(2: 1)$ & 3.0 & $10 \mathrm{~h}$ & r.t & 80 \\
$\mathrm{Me}_{2} \mathrm{CO}: \mathrm{H}_{2} \mathrm{O}(2: 1)$ & 3.0 & $15 \mathrm{~h}$ & r.t & 100 \\
1,4 dioxane: $\mathrm{H}_{2} \mathrm{O}(2: 1)$ & 3.0 & $20 \mathrm{~h}$ & r.t & 70 \\
Acetonitrile: $\mathrm{H}_{2} \mathrm{O}(2: 1)$ & 3.0 & $20 \mathrm{~h}$ & r.t & 80 \\
$\mathrm{H}_{2} \mathrm{O}$ & 3.0 & $1 \mathrm{~h}$ & rt & 35 \\
$\mathrm{H}_{2} \mathrm{O}$ & 3.0 & $1 \mathrm{~h}$ & $80^{\circ} \mathrm{C}$ & 100 \\
\hline
\end{tabular}

With the optimal reaction conditions in hand, different substituted of styrenes were then reacted with TICA in water, followed by $o$-phenylene diamines (2) in acetone (Scheme 1) or benzo[c] $[1,2,5]$ thiadiazole-4,5-diamine (3) in acetone (Scheme-2) afforded substituted regioisomers 
of phenylquinoxalines (3a-3i) and phenyl-[1,2,5]thiadiazolo[3,4-f]quinoxalines (5a-5f) with a combined yields. Formation of regioisomers depends on substituted $o$-phenylenediamines.

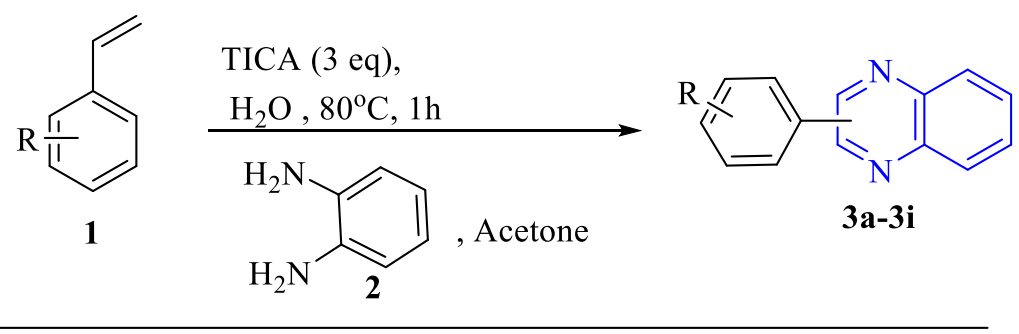<smiles>c1ccc(-c2cnc3ccccc3n2)cc1</smiles><smiles>Fc1ccc2c(c1)N=C[IH](c1ccccc1)=N2</smiles>

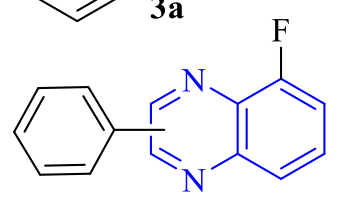<smiles>Fc1c(Br)ccc2nc(-c3ccccc3)ncc12</smiles><smiles>Cc1cccc(-c2cnc3ccccc3n2)c1</smiles>

3d<smiles>FC(F)(F)c1cccc(-c2cnc3cc(I)ccc3n2)c1</smiles>

$3 g$ $3 e$<smiles>Brc1ccc2nc(-c3ccccc3)ncc2c1</smiles>

$3 h$<smiles>FC(F)(F)c1cccc(-c2cnc3ccccc3n2)c1</smiles>

Scheme 1. Synthesis of substituted derivatives (3a-3i)

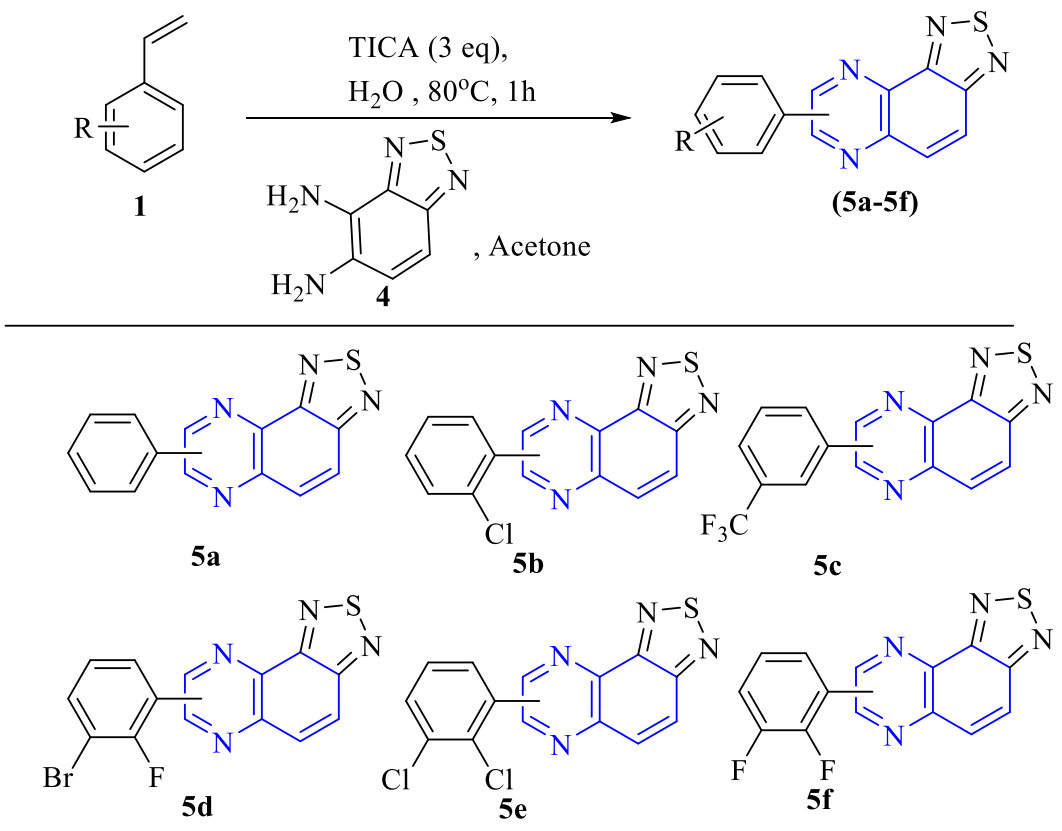

Scheme 2. Synthesis of substituted derivatives (5a-5f) 


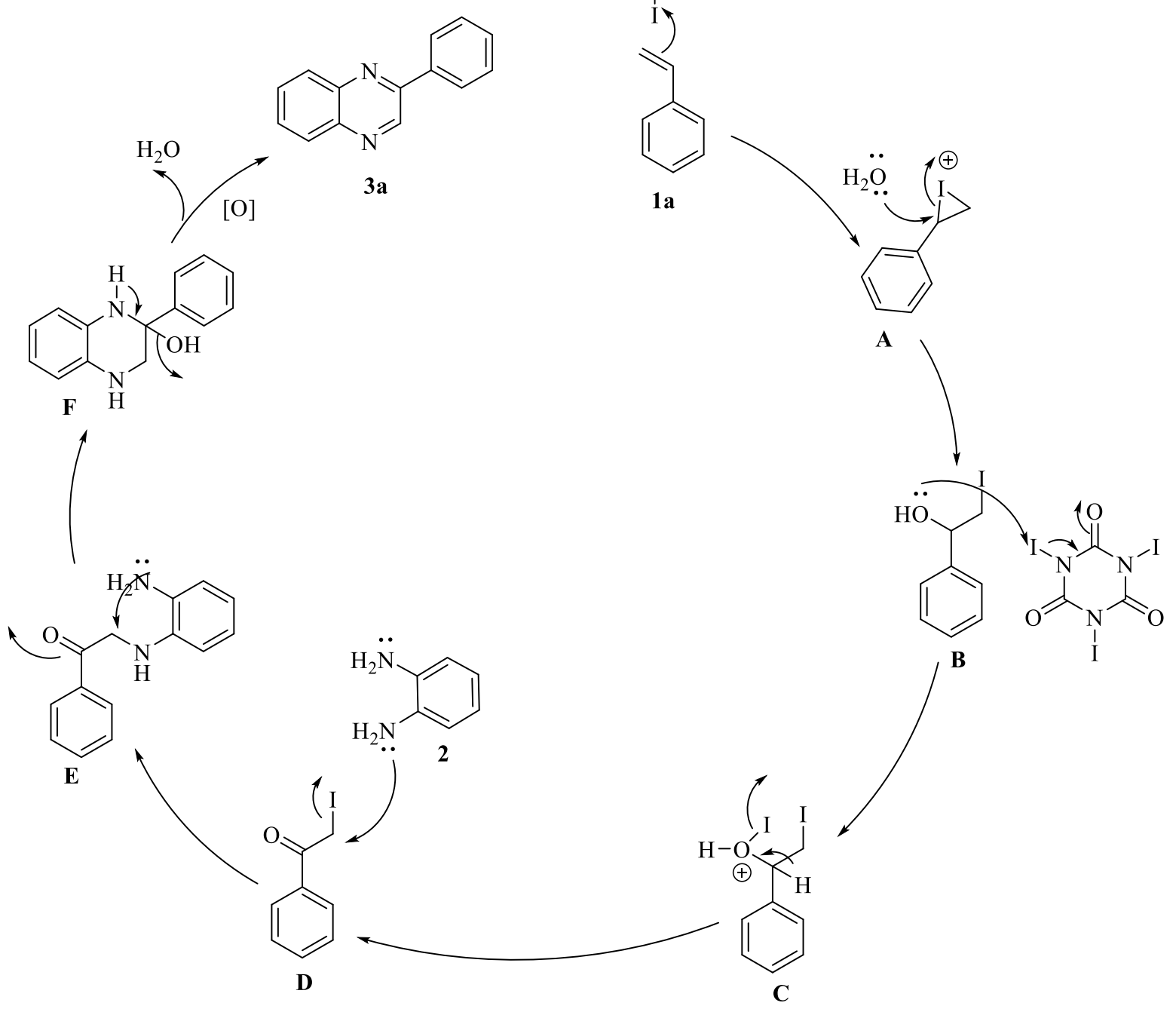

Figure 2. Proposed mechanism

Under the optimized reaction conditions, the substrate scope and versatility of the reaction was subsequently explored, and the results are summarized in Scheme-1 and Scheme 2. To our delight, the protocol was companionable with $o$-phenylenediame and styrenes containing a range of functional groups, little influence of electronic properties was observed on their reactivity. For example, $o$ phenylenediamine with electron-withdrawing group, such as $\mathrm{F}$ and styrene with electron-withdrawing group, such as $\mathrm{CF}_{3}$, yielded the corresponding product in $79 \%$ (Scheme 1, compound $\mathbf{3 g}$ ). On the other hand, o-phenylenediamne bearing electron-withdrawing groups such as $\mathrm{F}, \mathrm{Cl}$ also gave the desired product with a yields $76 \%$ to $74 \%$ respectively (Scheme-1, entries $\mathbf{3 b}$ and $\mathbf{3 c}$ ). Results from scheme2 , styrene with electron-withdrawing group, such as $\mathrm{CF}_{3}$, yielded the corresponding product in $75 \%$ (Scheme-2, compound 5c). Based on the literature and our previous work, we have proposed a plausible mechanism depicted in Figure 2. Based on the literature ${ }^{24}$, we have proposed a plausible mechanism depicted in Figure 2. In presence of TICA, styrene (1a) forms cyclic iodonium ion (A) which undergoes a ring opening through nucleophilic attack of $\mathrm{H}_{2} \mathrm{O}$ to give bromohydrin (B) as an intermediate. Bromohydrin (B) in the presence of TICA undergoes oxidation via (C) to form $\alpha$ iodoacetophenone (D). o-phenylenediamine was reacted with intermediate (D) via $(\mathbf{E}$ and $\mathbf{F})$ forms quinoxaline (3a). 


\section{Conclusions}

In summary, we have reported a triiodoisocyanuric acid promoted method for synthesis of key intermediate $\alpha$-bromo ketone via co-bromination and oxidation for the formation of an intermediate in the presence of triiodoisocyanuric acid, followed by condensation with the o-phenylenediamine (2) and benzo[c][1,2,5]thiadiazole-4,5-diamine (4) for the synthesis of phenylquinoxalines $(\mathbf{3 a - 3 i})$ and phenyl-[1,2,5]thiadiazolo[3,4-f]quinoxaline (5a-5f) in 55-79\% yield. This protocol is simple, broad substrate scope, good to excellent yields. We strongly believe that this protocol will serve as an encroachment to the existing methods and will be widely adopted for the synthesis of biologically important quinoxalines.

\section{Acknowledgement}

We thank to the Council of Scientific and Industrial Research (CSIR), New Delhi, India.

\section{Conflict of Interest}

The authors declare that they have no conflict of interest

\section{Supporting Information}

Supporting information accompanies this paper on http://www.acgpubs.org/journal/organic$\underline{\text { communications }}$

ORCID

Suresh Kuarm Bowroju: 0000-0001-9906-0625

Hanumaiah Marumamula: 0000-0002-7352-8477

Rajitha Bavanthula: 0000-0003-4011-0354

\section{References}

[1] Antoniotti, S.; Dunach, E. Direct and catalytic synthesis of quinoxaline derivatives from epoxides and ene-1,2diamines. Tetrahedron Lett. 2002, 43, 3971-3973.

[2] Guillon, J.; Grellier, P.; Labaied, M.; Sonnet, P.;Leger, J.M.; Deprez-Poulain, R.; Forfar- Bares, I.; Dallemagne, P.; Lemaitre, N.; Pehourcq, F.; Rochette, J.; Sergheraert, C.; Jarry, C. Synthesis, Antimalarial activity, and molecular modeling of new pyrrolo[1,2-a]quinoxalines, bispyrrolo[1,2-a]quinoxalines, vispyrido[3,2-e]pyrrolo[1,2-a]pyrazines, and bispyrrolo[1,2-a]thieno[3,2-e]pyrazines. J. Med. Chem. 2004, 47,1997-2009.

[3] Alleca, S.; Corona, P.; Loriga, M.; Paglietti, G.; Loddo, R.; Mascia, V.; Busonera, B.; La C. Quinoxaline chemistry. Part 16. 4-Substituted anilino and 4-substituted phenoxymethyl pyrrolo[1,2-a]quinoxalines and $N$ [4-(pyrrolo[1,2-a]quinoxalin-4-yl)amino and hydroxymethyl]benzoyl glutamates. Synthesis and evaluation of in vitro biological activity. Farmaco 2003, 58, 639-650.

[4] Desplat, V.; Geneste, A.; Begorre, M.-A.; Fabre, S. B.; Brajot, S.; Massip, S.; Thiolat, D.; Mossalayi, D.; Jarry, C.; Guillon, J. Synthesis of new pyrrolo[1,2-a]quinoxaline derivatives as potential inhibitors of akt kinase. $J$. Enzyme Inhib. Med. Chem. 2008, 23, 648-658.

[5] Desplat, V.; Moreau, S.; Gay, A.; Fabre, S. B.; Thiolat, D.; Massip, S.; Macky, G.; Godde, F.; Mossalayi, D.; Jarry, C.; Guillon, J. Synthesis and evaluation of the antiproliferative activity of novel pyrrolo[1,2a]quinoxaline derivatives, potential inhibitors of akt kinase. Part II. J. Enzyme Inhib. Med. Chem. 2010, 25, 204-215.

[6] Guillon, J.; Forfar, I.; Mamani-Matsuda, M.; Desplat, V.; SaliHge, M.; Thiolat, D.; Massip, S.; Tabourier, A.; Liger, J.-M.; Dufaure, B.; Haumont, G.; Jarry, C.; Mossalayi, D. Synthesis, analytical behaviour and biological evaluation of new 4-substituted pyrrolo[1,2-a]quinoxalines as antileishmanial agents. Bioorg. Med. Chem. 2007, 15, 194-210. 
[7] Gemma, S.; Colombo, L.; Forloni, G.; Savini, L.; Fracasso, C.; Caccia, S.; Salmona, M.; Brindisi, M.; Joshi, B. P.; Tripaldi, P.; Giorgi, G.; Taglialatela-Scafati, O.; Novellino, E.; Fiorini, I.; Campiani, G.; Butini, S. Pyrroloquinoxalinehydrazones as fluorescent probes for amyloid fibrils. Org. Biomol. Chem. 2011, 9, 51375148.

[8] Antoniotti, S.; Donach, E. Direct and catalytic synthesis of quinoxaline derivatives from epoxides and ene1,2-diamines. Tetrahedron Lett. 2002, 43, 3971-3973.

[9] Raw, S.A.; Wilfred, C.D.; Taylor R.J.K. Tandem oxidation processes for the preparation of nitrogencontaining heteroaromatic and heterocyclic compounds. Org. Biomol. Chem. 2004, 2, 788-796.

[10] Venkatesh, C.; Singh, B.; Mahata, P.K.; Ila, H.; Junjappa, H. Heteroannulation of nitroketene N,Sarylaminoacetals with $\mathrm{POCl}_{3}$ : A novel highly regioselective synthesis of unsymmetrical 2,3-substituted quinoxalines. Org. Lett. 2005, 7, 2169-2172.

[11] More, S.V.; Sastry, M.N.V.; Yao, C.F. Cerium (iv) ammonium nitrate (CAN) as a catalyst in tap water: A simple, proficient and green approach for the synthesis of quinoxalines. Green. Chem. 2006, 8, 91-95.

[12] Rajesh, S.B.; Swapnil, R.S.; Suresh, S.A.; Wamanrao, N.J.; Sudhakar, R.B.; Rajendra, P. An efficient protocol for the synthesis of quinoxaline derivatives at room temperature using molecular iodine as the catalyst. Tetrahedron Lett. 2005, 46, 7183-7186.

[13] Huang, T.; Wang, R.; Shi, L.; Lu, X. Montmorillonite K-10: An efficient and reusable catalyst for the synthesis of quinoxaline derivatives in water. Catal. Commun. 2008, 9, p.1143.

[14] Cai, J.J.; Zou, J.P.; Pan, X.Q.; Zhang, W. Gallium(III) triflate-catalyzed synthesis of quinoxaline derivatives. Tetrahedron Lett. 2008, 49, 7386-7390.

[15] Nandi, G.C.; Samai, S.; Kumar, R.; Singh, M.S. Silica-gel-catalyzed efficient synthesis of quinoxaline derivative under solvent-free conditions. Synth. Commun. 2011, 41, 417- 425.

[16] Mahesh, R.; Dhar, A.K.; Sasank, T.T.; Thirunavukkarasu, S.; Devadoss, T. Citric acid: An efficient and green catalyst for rapid one pot synthesis of quinoxaline derivatives at room temperature. Chinese Chem. Lett. 2011, 22, 389-392.

[17] Hasaninejad, A.; Zare, A.; Mohammadizadeh, M.R.; Shekouhya M. Oxalic acid as an efficient, cheap, and reusable catalyst for the preparation of quinoxalines via condensation of 1,2-diamines with $\alpha$-diketones at room temperature. Arkivoc. 2008, 13, 28-35.

[18] Krishnakumar, B.; Velmurugan, R.; Jothivel, S.; Swaminathan, M. An efficient protocol for the green synthesis of quinoxaline and dipyridophenazine derivatives at room temperature using sulfated titania. Catal. Commun. 2010, 11, 997-1002.

[19] Krishnakumar, B.; Swaminathan, M.J. A recyclable and highly effective sulfated $\mathrm{TiO}_{2}-\mathrm{P} 25$ for the synthesis of quinoxaline and dipyridophenazine derivatives at room temperature. Organomet. Chem. 2010, 695, 25722577.

[20] Chao, C.S.; Oh, S.G. Copper-catalyzed oxidative cyclization of $\alpha$-hydroxyketones with $o$ phenylenediamines leading to quinoxalines. J. Mol. Catal A. 2007, 276, 205-210.

[21] Kuarm, B.S.; Crooks, Peter. A.; Rajitha, B. An expeditious synthesis of quinoxalines by using biodegradable cellulose sulfuric acid as a solid acid catalyst. Green. Chem. Lett. Rev. 2013, 3, 228-232.

[22] Andrade de, V.S.C.; de Mattos, M.C.S. N-Halo Reagents: Modern synthetic approaches for heterocyclic synthesis. Synthesis 2019, 51, 1841-1870.

[23] Lyubchuk, T.V.; Hordiyenko, O.V. The use of $N$-halosuccinimides for cyclization with the formation of five-membered heterocyclic compounds. Chem. Het.. Compd. 2020, 56, 1-29.

[24] Mendonça, G.F.; de Mattos, M.C.S. Green chlorination of organic compounds using trichloroisocyanuric acid (TCCA). Curr. Org. Synth. 2013, 10, 820-822.

[25] de Almeida, L.S.; Esteves, P.M.; de Mattos, M.C.S. Tribromoisocyanuric acid: A green and versatile reagent Curr. Green Chem. 2014, 1, 94-107.

[26] Mendonça, G.F.; de Almeida, L.S.; de Mattos, M.C.S.; Esteves, P.M.; Ribeiro, R.S. New ideas for a 160 years-old reaction. Curr. Org. Synth. 2015, 12, 603-617.

[27] Tozetti, S.D.F.; de Almeida, L.S.; Esteves, P.M.; de Mattos, M.C.S. Trihaloisocyanuric acids/NaX: an environmentaly friendly system for vicinal dihalogenation of alkenes without using molecular halogen. $J$. Braz. Chem. Soc. 2007, 18, 675-677.

[28] Venu Madhav, J.; Thirupathi Reddy, Y.; Narsimha Reddy, P.; Nikhil Reddy, M.; Kuarm. S.; Crooks, A.; Rajitha, B. Cellulose sulfuric acid: An efficient biodegradable and recyclable solid acid catalyst for the onepot synthesis of aryl-14H-dibenzo[a.j]xanthenes under solvent-free conditions. J. Mol. Catal. A. 2009, 304, 85-87.

[29] B. Kuarm. B. S.; Rajitha, B. Xanthan sulfuric acid: An efficient, biosupported, and recyclable solid acid catalyst for the synthesis of 4,4'-(arylmethylene)bis(1H-pyrazol-5-ols). Synth. Commun. 2012,42, 2382-2387.

[30] Kuarm. B. S.; Janardan.B.; Rajitha, B. Xanthan sulfuric Acid: An efficient bio-supported and recyclable solid acid catalyst for the synthesis of 2-aryl thiadiazolo benzimidazoles. Chinese J. Chem. 2012, 30, 947950. 
[31] B Kuarm. B. S.; Venu Madhav, J.; Laxmi, S.L.; Rajitha, B.; Thirupathi Reddy, Y.; Narsimha Reddy, P.; Crooks, A. Cellulose sulfuric acid: An efficient biodegradable and recyclable solid acid catalyst for the synthesis of 1-oxo-hexahydroxanthene. Synth. Commun. 2011, 41, 1719-1724.

[32] Koca, I.; Yildirim,I. Synthesis and spectroscopic characterization of pyrrole-2,3- diones and their following reactions with 1,2-aromatic diamines. Org. Commun. 2012, 5, 135-142.

[33] A. Cellulose sulfuric acid: Novel and efficient biodegradable and recyclable acid catalyst for the solid-state synthesis of thiadiazolo benzimidazoles. Synth. Commun. 2011, 41, 662-669.

[34] Suresh, B.K.; Venu Madhav, J.; Rajitha, B. Synthesis and antimicrobial studies of selenadiazolo benzimidazoles. J. Het. Chem. 2013, 50, 1337-1341.

[35] Suresh, B.K.; Venu Madhav, J.; Rajitha, B. Xanthan sulfuric acid: An efficient and recyclable solid acid catalyst for Pechmann condensation. Synth. Commun. 2012, 42, 1770-1777.

[36] Kuarm, B. S.; Venu Madhav, J.; Laxmi, S.L.; Rajitha, B. Xanthan sulfuric acid: A new and efficient biosupported solid acid catalyst for the synthesis of 3,4-dihydropyrimidin-2(1H)-ones. Synth. Commun. 2012, 42, 1211-1217.

[37] Suresh, B.K.; Venu Madhav, J.; Laxmi, S.L.; Rajitha, B. Thirupathi Reddy, Y.; Narsimha Reddy, P.; Crooks, A. Expeditious Pechmann condensation by using biodegradable cellulose sulfuric acid as a solid acid catalyst. Synth. Commun. 2010, 40, 3358-3364.

[38] Kuarm, B. S.; Venu Madhav, J.; Rajitha, B. Xanthan sulfuric acid: An efficient bio-supported and recyclable solid acid catalyst for the synthesis of 2-aminothiazole-5-carboxylates and 2-aminoselenazole-5carboxylates. Lett. In Org. Chem. 2011, 8, 549-553.

[39] Venu Madhav, B. Suresh, B.K.; Thirupathi Reddy, Y.; Crooks, A. $\mathrm{CuPy}_{2} \mathrm{Cl}_{2}$ : A novel and efficient catalyst for synthesis of propargylamines under the conventional method and microwave irradiation. Synth. Commun. 2008, 38, 3215-3223.

[40] Venu Madhav, J.; Kuarm. B. S.; Rajitha, B. Solid-state synthesis of 1,3-selenazoles employing $\mathrm{CuPy}_{2} \mathrm{Cl}_{2}$ as a Lewis acid catalyst. Synth. Commun. 2008, 38, 3514-3522.

[41] Rajitha, B. Suresh, B.K.; Thirupathi Reddy, Y.; Crooks, A. Dipyridine cobalt chloride: A novel catalyst for the synthesis of coumarins via Pechmann condensation. Chem. Res. 2008, 4, 232-234.

[42] Kuarm, B.S.; Reddy, Y.T.; Venumadhav, J.; Crooks, P.A.; Rajitha, B. 3-[Benzimidazo- and 3[benzothiadiazoleimidazo-(1,2-c)quinazolin-5-yl]-2H-chromene-2-ones as potent antimicrobial agents. Bio. Med. Chem. Lett. 2011, 21, 524-527.

[43] Venu Madhav, J.; Kuarm. B. S.; Rajitha, B. Dipyridine cobalt chloride: a novel and efficient catalyst for the synthesis of 14-aryl 14H-dibenzo[a.j]xanthenes under solvent-free condition. Arkivoc 2008, 2, 204-209.

[44] Venu Madhav, J.; Kuarm. B. S.; Rajitha, B. Dipyridine copper chloride as a mild and efficient catalyst for the solid state synthesis of 2- substituted benzimidazoles. Arkivoc 2008. 13,145-150.

[45] Kumar, V.; Venu Madhav, J.; Kuarm. B. S.; Rajitha, B. Synthesis of 3-[6-(2-amino-ethoxy)-3-methyl-5-(3morpholin-4-yl-propionyl)-benzofuran-2 carbonyl]-chromen-2-one under microwave irradiation conditions. Green. Chem. Lett. Rev. 2011, 4, 97-101.

[46] Suresh, K. B.; Crooks, P.A.; Rajitha, B. Xanthan sulfuric acid: An efficient biodegradable solid acid catalyst for the synthesis of 14-aryl-14H-dibenzo [a, i] xanthene-8, 13-diones. Adv. Appl. Sci. Res. 2012, 3, 1-5.

[47] Suresh, K. B.; Crooks, P.A.; Rajitha, B. Polyvinylsulfonic acid: An efficient and recyclable Bronsted acid catalyst for Pechmann condensation. Int. J. Res. Pharm. Biomed. Sci 2012, 3, 50-53.

[48] Laxmi, S. V.; Kuarm, B.S.; Rajitha, B. Synthesis and antimicrobial activity of coumarin pyrazole pyrimidine 2, 4, $6(1 \mathrm{H}, 3 \mathrm{H}, 5 \mathrm{H})$ triones and thioxopyrimidine4, $6(1 \mathrm{H}, 5 \mathrm{H})$ diones. Med. Chem. Res., 2013, 22, 768-774.

[49] Laxmi, S. V.; Reddy, Y.T.; Kuarm, B.S. Reddy, P.N.; Crooks, P.A.; Rajitha, B. Synthesis and evaluation of chromenyl barbiturates and thiobarbiturates as potential antitubercular agents. Bioorg. Med. Chem. Lett. 2011, 21(14), 4329-4331.

[50] Rodrigo da, S. R.; Pierre, M. E.; Marcio, M. Triiodoisocyanuric acid: a new and convenient reagent for regioselective coiodination of alkenes and enolethers with oxygenated nucleophiles. Tetrahedron Lett. 2007, 48, 8747-8751.

[51] Vitor, A.; Marcio, M. One-pot synthesis of 4-aryl-2-aminothiazoles from styrenes and thioureas promoted by tribromoisocyanuric acid. Tetrahedron Lett. 2020, 61, 152-164.

[52] Soltani, M.; Mohammadpoor-Baltork, I.; Khosropour, A.R. Majid, M.; Shahram, T.; Valiollah M. Efficient synthesis of 2-arylindoles, 2-arylimidazo[1,2-a]pyridines and 2-arylquinoxalines, and their bis-derivatives using [Hmim] OTf ionic liquid supported on nano-silica as a reusable catalyst. J. Iran.Chem.Soc. 2015, 12, 1369-1380.

[53] Shao-Jie, L.; Dan-Qian, X.; Ai-Bao, Xia.; Yi-Feng, Wang.; Yun-Kui, L.; Xiao-Hua, Du.; Zhen-Yuan, Xu. $\mathrm{Pd}(\mathrm{OAc})_{2}$-catalyzed regioselective aromatic $\mathrm{C}-\mathrm{H}$ bond fluorination. Chem. Commun. 2013, 49, 6218 - 6220. 
[54] Dong, Z.; Clososki, G. C.; Wunderlich, S. H.; Unsinn, A.; Li, J.; Knochel, Direct zincation of functionalized aromatics and heterocycles by using a magnesium base in the presence of $\mathrm{ZnCl}_{2}$. Chem. Eur. J. 2009, 5, 457468.

[55] Pardeshi, S. D.; Sathe, A.; Vadagaonkar, K. S.; Chaskar, A. C. One-pot protocol for the synthesis of imidazoles and quinoxalines using N-Bromosuccinimide. Adv. Synth. Catal. 2017, 359, 4217 - 4226.

\section{A C G \\ publications \\ (C) 2021 ACG Publications}

